\title{
Signal Recovery from Low Frequency Components
}

\author{
Yonina C. Eldar \\ Department of Electrical Engineering \\ Technion - Israel Institute of Technology \\ Haifa 32000, Israel \\ e-mail: yonina@ee.technion.ac.il
}

\begin{abstract}
In many applications only the low frequency components of a signal can be measured due to the lowpass behavior of many physical systems. Nevertheless, if additional information on the structure of the signal is known, it might still be possible to reconstruct the signal from its low-frequency content. This paper studies signals in shift-invariant spaces with multiple generators and derives necessary conditions on the bandwith of the lowpass filter as well as sufficient conditions on the generators such that signal recovery is possible. If the signal can not be recovered from its low frequency components, an appropriate pre-processing of the signal is proposed which improves the reconstruction ability. In particular, it is shown that modulating the signal with one or more mixing functions prior to lowpass filtering can ensure the recovery of the signal in many cases.
\end{abstract}

\section{INTRODUCTION}

Lowpass filters are prevalent in biological, physical and engineering systems such that in many scenarios, we do not have access to the entire frequency content of a signal we wish to process, but only to its low frequencies. In this paper, we study in detail under what conditions a signal $x$ can be recovered from its low-frequency content. Clearly if we have no prior knowledge on the original signal, and we are given a lowpassed version of it, then we cannot recover the missing frequency content. However, if some knowledge on the signal structure is available then it may be possible to interpolate it from the given data. Our focus here is on signals that lie in shift-invariant (SI) spaces, generated by multiple generators [1]-[3]. For these signals, we derive a necessary condition on the cutoff frequency of the low pass filter (LPF) and sufficient conditions on the generators such that $x$ can be recovered from its lowpassed version. If these recovery conditions are satisfied, a concrete method to reconstruct $x$ from its lowpass frequency content is provided.

As expected, there are scenarios in which recovery is not possible. For these cases, we investigate whether we can improve our ability to determine the signal by appropriate pre-processing. In particular, we consider the situation where the signal $x$ is modulated by multiplying it with a periodic mixing function prior to lowpass filtering. We then derive conditions on the mixing function to ensure perfect recovery. As we show, a larger class of signals can be recovered this way. Moreover, by applying a bank of mixing functions, the necessary cutoff frequency in each channel can be reduced. Finally, we briefly discuss how the results we developed can be applied to sampling sparse signals in SI spaces at rates

\author{
Volker Pohl \\ Department of Electrical Engineering and \\ Computer Science, Technische Universität Berlin \\ 10587 Berlin, Germany \\ e-mail: volker.pohl@mk.tu-berlin.de
}

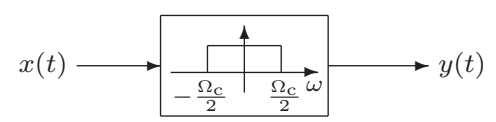

lower than Nyquist. These ideas rely on the recently developed framework for analog compressed sensing [4]-[7]. In our setting, they translate to reducing the LPF bandwidth, or the number of modulators.

Because of the limited space we will not give proofs of the presented results, but we refer to [8] for detailed proofs and for some more concrete examples.

\section{Problem Formulation}

As usual, $\mathbb{C}^{N}, L^{2}$, and $\ell^{2}$ denote the $N$-dimensional Euclidean space, the space of square integrable function on the real line, and the space of square summable sequences, respectively. All these spaces are Hilbert spaces with the usual inner products. Throughout the paper

$$
\widehat{x}(\omega)=\int_{\mathbb{R}} x(t) \mathrm{e}^{-\mathrm{i} \omega t} \mathrm{~d} t, \quad \omega \in \mathbb{R},
$$

denotes the Fourier transform of a function $x \in L^{2}$, and for any $T \in \mathbb{R}$, the translation operator $\mathrm{S}_{T}: L^{2} \rightarrow L^{2}$ is defined by $\left(\mathrm{S}_{T} x\right)(t)=x(t-a)$.

We consider the problem of recovering a signal $x \in L^{2}$ from its low-frequency content. Specifically, suppose that $x$ is filtered by a LPF with cut-off frequency $\Omega_{\mathrm{c}} / 2$, as in Fig. 1:

$$
y(t)=\left(\mathrm{P}_{\Omega_{\mathrm{c}}} x\right)(t)=\frac{1}{2 \pi} \int_{-\Omega_{\mathrm{c}} / 2}^{\Omega_{\mathrm{c}} / 2} \widehat{x}(\omega) \mathrm{e}^{\mathrm{i} \omega t} \mathrm{~d} \omega .
$$

We are going to study the following two questions:

1) What signals $x$ can be recovered from the LPF output $y$ ?

2) Can we perform preprocessing of $x$ prior to filtering to ensure that $x$ can be recovered from $y$ ?

It is well known that if $x$ is bandlimited to $\left[-\Omega_{\mathrm{c}} / 2, \Omega_{\mathrm{c}} / 2\right]$, then it can be recovered from $y$. However, we assume here that $x$ lies in a general SI subspace of $L^{2}$, and so it is not necessarily bandlimited. More precisely, let $\phi=\left\{\phi_{1}, \ldots, \phi_{N}\right\}$ be a given set of functions in $L^{2}$ and let $T \in \mathbb{R}$ be a real number. Then the shift-invariant space generated by $\phi$ is defined as [1]-[3]

$$
\mathcal{S}_{T}(\phi)=\overline{\operatorname{span}}\left\{\mathrm{S}_{T}^{k} \phi_{n}: k \in \mathbb{Z} ; n=1, \ldots, N\right\}
$$


and where the functions $\phi_{n}$ are referred to as the generators of $\mathcal{S}_{T}(\phi)$. Thus, every function $x \in \mathcal{S}_{T}(\phi)$ can be written as

$$
x(t)=\sum_{n=1}^{N} \sum_{k \in \mathbb{Z}} a_{n}[k]\left(\mathrm{S}_{T}^{k} \phi_{n}\right)(t), \quad t \in \mathbb{R},
$$

with certain coefficient sequences $\boldsymbol{a}_{n}=\left\{a_{n}[k]\right\}_{k \in \mathbb{Z}}$ for every $n=1, \ldots, N$. The number $R_{I}=N / T$ associated with $\mathcal{S}_{T}(\phi)$ is usually be called the rate of innovation [9]. To guarantee a unique and stable representation of every signal in $\mathcal{S}_{T}(\phi)$ in terms of the coefficient sequences $\boldsymbol{a}_{n}$, the generators $\phi$ and the shift parameter $T$ are typically chosen such that the sequence $\phi:=\left\{\mathrm{S}_{T}^{k} \phi_{n}\right\}_{k \in \mathbb{Z}}^{n=1, \ldots, N}$ forms a Riesz basis for $\mathcal{S}_{T}(\phi)$. This is the case if there exist constants $0<A_{\phi} \leq B_{\phi}<\infty$ (the so called frame bounds) such that

$$
A_{\phi}\|\boldsymbol{a}\|_{\ell^{2}}^{2} \leq\left\|\sum_{n=1}^{N} \sum_{k \in \mathbb{Z}} a_{n}[k] \mathrm{S}_{T}^{k} \phi_{n}\right\|_{L^{2}}^{2} \leq B_{\phi}\|\boldsymbol{a}\|_{\ell^{2}}^{2},
$$

for every sequence $\boldsymbol{a}=\left\{a_{n}[k]\right\}_{k \in \mathbb{Z}}^{n=1, \ldots, N}$ in $\ell^{2}$. Condition (2) implies that for every $x \in \mathcal{S}_{T}(\phi)$, the coefficient sequences $\boldsymbol{a}_{n}$ are in $\ell^{2}$, and conversely that every $x \in \mathcal{S}_{T}(\phi)$ is uniquely determined by some sequences $\boldsymbol{a}_{n} \in \ell^{2}$. In particular, (2) guarantees that the coefficient sequences $\boldsymbol{a}_{n}$ can be recovered from $x \in \mathcal{S}_{T}(\phi)$ by means of a linear bounded operator.

Condition (2) can be reformulated. To this end, we associate with every sequence in $L^{2}$ of the form $\phi:=\left\{\mathrm{S}_{T}^{k} \phi_{n}\right\}_{k \in \mathbb{Z}}^{n=1, \ldots, N}$ the matrix $\boldsymbol{\Phi}(\omega)$ with $N$ columns and infinity many rows whose entry in the $k$ th row and $n$th column is

$$
[\boldsymbol{\Phi}(\omega)]_{k, n}=\widehat{\phi}^{(n)}(\omega+k \Omega), \quad \omega \in[-\Omega / 2, \Omega / 2]
$$

and where $\Omega:=2 \pi / T$. Moreover, the $N \times N$ matrix

$$
\mathbf{G}_{\phi}(\omega):=\frac{1}{T} \boldsymbol{\Phi}(\omega)^{*} \boldsymbol{\Phi}(\omega)
$$

is called the Grammian associated with the sequence $\phi$. Therewith, we can reformulate the condition for $\phi$ to be a Riesz basis. Namely [2], $\phi$ is a Riesz basis with frame bounds $A_{\phi}, B_{\phi}$ if and only if

$$
A_{\phi} \mathbf{I}_{N} \preceq \mathbf{G}_{\phi}(\omega) \preceq B_{\phi} \mathbf{I}_{N}, \quad \text { a.e. } \omega \in[-\Omega / 2, \Omega / 2] .
$$

Examples of SI spaces include multiband signals [10] and spline functions [11], [12]. Expansions of the type (1) are also encountered in communication systems, when the analog signal is produced by pulse amplitude modulation.

\section{RECOVERY CONDITIONS}

The first question we address is whether we can recover $x \in \mathcal{S}_{T}(\phi)$ of the form (1) from the output $y=\mathrm{P}_{\Omega_{\mathrm{c}}} x$ of a LPF with bandwidth $\Omega_{\mathrm{c}}$, assuming that the generators $\phi$ satisfy (4). To recover $x \in \mathcal{S}_{T}(\phi)$ it is sufficient to recover the sequences $\boldsymbol{a}_{n}=\left\{a_{n}[k]\right\}_{k \in \mathbb{Z}}$ because the generators $\phi_{n}$ are assumed to be known. Since $\mathrm{P}_{\Omega_{\mathrm{c}}} \mathrm{S}_{T}=\mathrm{S}_{T} \mathrm{P}_{\Omega_{\mathrm{c}}}$ and because both operators are bounded, the output of the LPF can be written as

$$
y(t)=\left(\mathrm{P}_{\Omega_{\mathrm{c}}} x\right)(t)=\sum_{n=1}^{N} \sum_{k \in \mathbb{Z}} a_{n}[k]\left(\mathrm{S}_{T}^{k} \psi_{n}\right)(t)
$$

where $\psi_{n}:=\mathrm{P}_{\Omega_{\mathrm{c}}} \phi_{n}$ denotes the lowpass filtered generator $\phi_{n}$. This shows that $y \in \mathcal{S}_{T}(\psi)$ lies in the SI space spanned by the functions $\psi=\left\{\psi_{1}, \ldots, \psi_{N}\right\}$. Consequently, the coefficient sequences $\boldsymbol{a}_{n}, n=1, \ldots, N$ can be recovered from $y$ if $\boldsymbol{\psi}=\left\{\mathrm{S}_{T}^{k} \psi_{n}\right\}_{k, n}$ forms a Riesz basis for $\mathcal{S}_{T}(\psi)$, i.e. if the corresponding Grammian $\mathbf{G}_{\psi}(\omega)$ satisfies (4) for some $0<A_{\psi} \leq B_{\psi}<\infty$. Whether this is true depends on the bandwidth $\Omega_{\mathrm{c}}$ of the LPF and on the generators $\phi$. Next we give a necessary condition on the LPF bandwidth $\Omega_{\mathrm{c}}$ such that signal recovery is possible.

Proposition 1: Let $\mathcal{S}_{T}(\phi)$ be a SI space generated by $N$ functions $\phi=\left\{\phi_{1}, \ldots, \phi_{N}\right\}$ and set $\Omega:=2 \pi / T$. Then every $x \in \mathcal{S}_{T}(\phi)$ can be recovered from $y=\mathrm{P}_{\Omega_{\mathrm{c}}} x$ only if the $L P F$ bandwith is larger or equal than then the rate of innovation of $\mathcal{S}_{T}(\phi)$, i.e. only if

$$
\Omega_{\mathrm{c}} \geq N \Omega=N \frac{2 \pi}{T}=2 \pi R_{I}
$$

The above condition on the bandwidth $\Omega_{\mathrm{c}}$ of the LPF is not sufficient, in general. However, if $\Omega_{\mathrm{c}}$ satisfies (5), sufficient conditions on the generators $\phi$ can be derived such that the lowpass filtered generators $\psi$ generate a Riesz basis for $\mathcal{S}_{T}(\psi)$, i.e. such that $x$ can be recovered from $y$.

Proposition 2: Let $\phi=\left\{\phi_{1}, \ldots, \phi_{N}\right\}$ be the generators of $\mathcal{S}_{T}(\phi)$ and let $\Omega_{\mathrm{c}}$ be the bandwidth of the LPF. Denote by $L$ the smallest integer such that $\left(\Omega_{\mathrm{c}} / \Omega-1\right) / 2 \leq L$, and define the $(2 L+1) \times N$ matrix $\boldsymbol{\Phi}_{L}(\omega)$ by

$$
\left[\boldsymbol{\Phi}_{L}(\omega)\right]_{k, n}=\widehat{\phi}_{n}(\omega+k \Omega), \quad k=-L, \ldots, L .
$$

Then every $x \in \mathcal{S}_{T}(\phi)$ can be recovered from $y=\mathrm{P}_{\Omega_{\mathrm{c}}} x$ if there exists a constant $\alpha>0$ such that

$$
\mathbf{G}_{L}(\omega):=\frac{1}{T} \boldsymbol{\Phi}_{L}^{*}(\omega) \boldsymbol{\Phi}_{L}(\omega) \succeq \alpha \mathbf{I}_{N}
$$

for almost all $\omega \in[-\Omega / 2, \Omega / 2]$.

When $\Omega_{\mathrm{c}} \rightarrow \infty$ (i.e. $L \rightarrow \infty$ ) the matrix $\mathbf{G}_{L}(\omega)$ reduces to $\mathbf{G}_{\phi}(\omega)$ which by definition satisfies (4). However, since for the calculation of the entries of $\mathbf{G}_{L}(\omega)$ we are only summing over a partial set of the integers, we are no longer guaranteed that $\mathbf{G}_{L}(\omega)$ satisfies the lower bound of (4).

\section{SignAl RECOVERY}

We now describe a simple method to reconstruct the desired signal $x$ from its low frequency components. Throughout this section, it is assumed that the necessary and sufficient conditions of Prop. 1 and 2, respectively, are satisfied.

Taking the Fourier transform of (1), we see that every $x \in$ $\mathcal{S}_{T}(\phi)$ can be expressed in the Fourier domain as

$$
\widehat{x}(\omega)=\sum_{n=1}^{N} \widehat{a}_{n}\left(\mathrm{e}^{\mathrm{i} \omega T}\right) \widehat{\phi}_{n}(\omega)=\widehat{\phi}(\omega)^{\mathrm{T}} \widehat{\mathbf{a}}\left(\mathrm{e}^{\mathrm{i} \omega T}\right)
$$

where $\widehat{a}_{n}\left(\mathrm{e}^{\mathrm{i} \omega T}\right)=\sum_{k \in \mathbb{Z}} a_{n}[k] \mathrm{e}^{-\mathrm{i} \omega k T}$ is the $\Omega$-periodic discrete time Fourier transform of the sequence $\left\{a_{n}[k]\right\}_{k \in \mathbb{Z}}$, and where $\widehat{\mathbf{a}}\left(\mathrm{e}^{\mathrm{i} \omega T}\right)$ denotes the length $N$ vector whose $n$th element is equal to $\widehat{a}_{n}\left(\mathrm{e}^{\mathrm{i} \omega T}\right)$ and $\widehat{\phi}(\omega)$ denotes the vector whose $n$th element is equal to $\widehat{\phi}_{n}(\omega)$. The output $y=\mathrm{P}_{\Omega_{\mathrm{c}}} x$ of the LPF is bandlimited to $\omega \in\left[-\Omega_{\mathrm{c}} / 2, \Omega_{\mathrm{c}} / 2\right]$ so that

$$
\widehat{y}(\omega)=\widehat{\phi}(\omega)^{\mathrm{T}} \widehat{\mathbf{a}}\left(\mathrm{e}^{\mathrm{i} \omega T}\right), \quad \omega \in\left[-\Omega_{\mathrm{c}} / 2, \Omega_{\mathrm{c}} / 2\right] .
$$




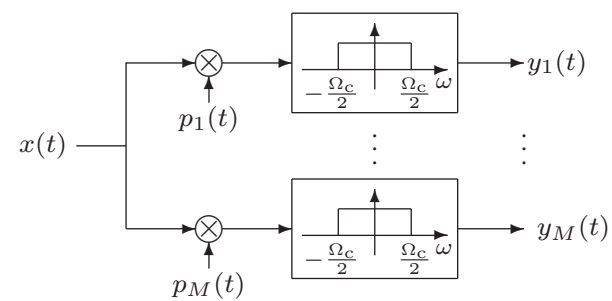

Fig. 2. Pre-processing with a bank of $M$ mixing functions.

For every $\omega \in\left[-\Omega_{\mathrm{c}} / 2, \Omega_{\mathrm{c}} / 2\right]$, (9) describes an equation for the $N$ unknowns $\widehat{a}_{n}\left(\mathrm{e}^{\mathrm{i} \omega T}\right)$. However, we need at least $N$ equations to recover the length- $N$ vector $\widehat{\mathbf{a}}\left(\mathrm{e}^{\mathrm{i} \omega T}\right)$. According to Prop. 1 the bandwidth of the LPF has to be at least $\Omega_{\mathrm{c}} \geq N \Omega$. Therefore we can form more equations from the given data by noting that $\widehat{a}$ is $\Omega$-periodic, while $\widehat{\phi}$, and consequently $\widehat{y}$, are generally not. Specifically, we have that $\widehat{\mathbf{a}}\left(\mathrm{e}^{\mathrm{i}[\omega+k \Omega] T}\right)=\widehat{\mathbf{a}}\left(\mathrm{e}^{\mathrm{i} \omega T}\right)$ for every $\omega \in[-\Omega / 2, \Omega / 2]$ and all $k \in \mathbb{Z}$. Consequently, by evaluating $\widehat{y}$ and $\widehat{\phi}$ at frequencies $-\Omega_{\mathrm{c}} / 2 \leq \omega_{k} \leq \Omega_{\mathrm{c}} / 2$, we can use (9) to generate more equations. To this end, let $L$ be defined as in Prop. 2. We then generate the equations

$$
\widehat{y}_{k}(\omega):=\widehat{y}(\omega-k \Omega)=\sum_{n=1}^{N} \widehat{\phi}_{n}(\omega-k \Omega) \widehat{a}_{n}(\omega-k \Omega)
$$

for $k=-L, \ldots, 0, \ldots, L$ and for $\omega \in[-\Omega / 2, \Omega / 2]$. All these observations are in the passband regime of the LPF. This set of $2 L+1$ equations may be written as

$$
\widehat{\mathbf{y}}(\omega)=\boldsymbol{\Phi}_{L}(\omega) \widehat{\mathbf{a}}\left(\mathrm{e}^{\mathrm{i} \omega T}\right), \quad \omega \in[-\Omega / 2, \Omega / 2],
$$

where $\widehat{\mathbf{y}}(\omega)=\left[\widehat{y}_{-L}(\omega), \ldots, 0, \ldots, \widehat{y}_{L}(\omega)\right]^{\mathrm{T}}$ is a length $2 L+1$ vector containing all the different observations $\widehat{y}_{k}$ of the output $\widehat{y}$, and $\Phi_{L}(\omega)$ is the $(2 L+1) \times N$ matrix given by (6).

If the matrix $\boldsymbol{\Phi}_{L}(\omega)$ satisfies the sufficient conditions of Prop. 2 then the unknown vector $\widehat{\mathbf{a}}\left(\mathrm{e}^{\mathrm{i} \omega T}\right)$ can be recovered from (10) by solving the linear set of equations for all $\omega \in$ $[-\Omega / 2, \Omega / 2]$. In particular, there exists a left inverse $\boldsymbol{\Phi}_{L}(\omega)^{-1}$ of $\boldsymbol{\Phi}_{L}(\omega)$ such that $\widehat{\mathbf{a}}\left(\mathrm{e}^{\mathrm{i} \omega T}\right)=\boldsymbol{\Phi}_{L}(\omega)^{-1} \widehat{\mathbf{y}}(\omega)$. Finally, the desired sequences $\left\{a_{n}[k]\right\}_{k \in \mathbb{Z}}$ are the Fourier coefficients of the $\Omega$ periodic functions $\widehat{a}_{n}$.

\section{Preprocessing of the Signal}

If the conditions of Prop. 2 are satisfied then every signal $x \in \mathcal{S}_{T}(\phi)$ can be recovered from $y=\mathrm{P}_{\Omega_{\mathrm{c}}} x$. Otherwise an interesting question is whether we can pre-process $x$ before lowpass filtering $(u=\mathrm{M} x)$ in order to ensure its recovery from the LPF output $y=\mathrm{P}_{\Omega_{\mathrm{c}}} u=\mathrm{P}_{\Omega_{\mathrm{c}}} \mathrm{M} x$. In particular we consider pre-processing of $x$ by multiplying it with a set of $T$-periodic mixing functions $p_{m}$ as shown as in Fig. 2:

$$
y_{m}(t)=\left(\mathrm{M}_{m} x\right)=p_{m}(t) x(t), \quad m=1, \ldots, M .
$$

\section{A. Single Channel $(M=1)$}

We begin with the case where the bank of mixers in Fig. 2 consist of only a single mixing function $p$ and a single output $y$. Since $p$ is assumed to be $T$-periodic, it can be written as

$$
p(t)=\sum_{k \in \mathbb{Z}} b_{k} \mathrm{e}^{\mathrm{i} 2 \pi k t / T}
$$

with the Fourier coefficients

$$
b_{k}=\frac{1}{T} \int_{-T / 2}^{T / 2} p(t) \mathrm{e}^{-\mathrm{i} 2 \pi k t / T} \mathrm{~d} t, \quad k \in \mathbb{Z} .
$$

The output $y=\mathrm{P}_{\Omega_{\mathrm{c}}}(p x)$ of the LPF is then given in the frequency domain by

$$
\widehat{y}(\omega)=\sum_{k \in \mathbb{Z}} b_{k} \widehat{x}(\omega-k \Omega), \quad \omega \in\left[-\Omega_{\mathrm{c}} / 2, \Omega_{\mathrm{c}} / 2\right] .
$$

Using (8) and the fact that $\widehat{a}_{n}\left(\mathrm{e}^{\mathrm{i} \omega T}\right)$ is $\Omega$-periodic, equation (13) can be rewritten as

$$
\widehat{y}(\omega)=\sum_{n=1}^{N} \widehat{a}_{n}\left(\mathrm{e}^{\mathrm{i} \omega T}\right) \sum_{k \in \mathbb{Z}} b_{k} \widehat{\phi}_{n}(\omega-k \Omega)
$$

with $\omega \in\left[-\Omega_{\mathrm{c}} / 2, \Omega_{\mathrm{c}} / 2\right]$. Defining

$$
\widehat{\gamma}_{n}(\omega):=\sum_{k \in \mathbb{Z}} b_{k} \widehat{\phi}_{n}(\omega-k \Omega), \quad n=1, \ldots, N
$$

and denoting by $\widehat{\gamma}$ the vector whose $n$th element is $\widehat{\gamma}_{n}$, we express (14) as

$$
\widehat{y}(\omega)=\widehat{\gamma}(\omega)^{\mathrm{T}} \widehat{\mathbf{a}}\left(\mathrm{e}^{\mathrm{i} \omega T}\right), \quad \omega \in\left[-\Omega_{\mathrm{c}} / 2, \Omega_{\mathrm{c}} / 2\right] .
$$

This equation is similar to (9) with $\widehat{\gamma}$ replacing $\widehat{\phi}$. Therefore, as in the case without pre-processing, we can create $2 L$ additional equations (where $L$ is defined as in Prop. 2) by evaluating $\widehat{y}(\omega)$ at frequencies $\omega+k \Omega$. This yields the system of equations

$$
\widehat{\mathbf{y}}(\omega)=\boldsymbol{\Gamma}_{L}(\omega) \widehat{\mathbf{a}}\left(\mathrm{e}^{\mathrm{i} \omega T}\right), \quad \omega \in[-\Omega / 2, \Omega / 2]
$$

with $\widehat{\mathbf{y}}$ and $\widehat{\mathbf{a}}$ are defined as in (10) and the $(2 L+1) \times N$ matrix $\Gamma_{L}(\omega)$ is given by

$$
\left[\boldsymbol{\Gamma}_{L}(\omega)\right]_{k, n}=\widehat{\gamma}_{n}(\omega+k \Omega), \quad k=-L, \ldots, 0, \ldots, L .
$$

So due to the mixing of the signal, the coefficient matrix $\boldsymbol{\Phi}_{L}(\omega)$ in (10) is changed to $\boldsymbol{\Gamma}_{L}(\omega)$ in (17). This new coefficient matrix is constructed out of the "new generators" $\left\{\gamma_{n}\right\}_{n=1}^{N}$ in exactly the same way as $\boldsymbol{\Phi}_{L}(\omega)$ is constructed from the original generators $\left\{\phi_{n}\right\}_{n=1}^{N}$. Consequently, $\widehat{\mathbf{a}}$ can be recovered from the given measurements as long as $\Gamma_{L}(\omega)$ has full column rank for all $\omega \in[-\Omega / 2, \Omega / 2]$, and it is not hard to see (cf. [8]) that to this end the LPF bandwidth has to satisfy again the necessary condition $\Omega_{\mathrm{c}} \geq N \Omega$ of Prop. 1 .

However, we now want to show that the sufficient conditions of Prop. 2 are easier to satisfy for $\boldsymbol{\Gamma}_{L}(\omega)$ than for the original matrix $\boldsymbol{\Phi}_{L}(\omega)$ given in (6). To this end, we write $\boldsymbol{\Gamma}_{L}(\omega)$ as

$$
\boldsymbol{\Gamma}_{L}(\omega)=\mathbf{B}_{L} \boldsymbol{\Phi}(\omega)
$$

where $\boldsymbol{\Phi}(\omega)$ is the matrix (3) with $N$ columns and infinite rows. The matrix $\mathbf{B}_{L}$ with $2 L+1$ rows and infinite columns contains the Fourier coefficients $\left\{b_{k}\right\}_{k \in \mathbb{Z}}$ of the mixing sequence (11). Its entry in the $n$th row and $m$ th column is

$$
\left[\mathbf{B}_{L}\right]_{n, m}=b_{m-n}, \quad n=-L, \ldots, 0, \ldots, L ; m \in \mathbb{Z} .
$$

Representation (18) follows immediately from the relation $\widehat{\gamma}_{n}(\omega-\ell \Omega)=\sum_{k \in \mathbb{Z}} b_{k-\ell} \widehat{\phi}_{n}(\omega-k \Omega)$ for the entries of the 
matrix $\Gamma_{L}(\omega)$. Due to the similarity with the case without preprocessing, it is clear that recovery from the LPF output $y$ will be possible if $\boldsymbol{\Gamma}_{L}(\omega)$ satisfies a condition like (7), i.e. the question is whether we can choose the sequence $\left\{b_{k}\right\}_{k \in \mathbb{Z}} \in \ell^{2}$, and consequently the mixing function $p$, so that the matrix $\boldsymbol{\Gamma}_{L}^{*}(\omega) \boldsymbol{\Gamma}_{L}(\omega)=\boldsymbol{\Phi}^{*}(\omega) \mathbf{B}_{L}^{*} \mathbf{B}_{L} \boldsymbol{\Phi}(\omega)$ is invertible for all $\omega \in[-\Omega / 2, \Omega / 2]$. If we choose $p(t) \equiv 1$ then $b_{0}=1$ and $b_{k}=0$ for all $k \neq 0$. Consequently $\mathbf{B}_{L} \boldsymbol{\Phi}(\omega)$ is comprised of the central $2 L+1$ rows of $\boldsymbol{\Phi}(\omega)$, so that $\boldsymbol{\Gamma}_{L}(\omega)=\boldsymbol{\Phi}_{L}(\omega)$. However, by allowing for general sequences $\left\{b_{k}\right\}_{k \in \mathbb{Z}}$, we have more freedom in choosing $\mathbf{B}_{L}$ such that $\mathbf{B}_{L} \boldsymbol{\Phi}(\omega)$ may have full column-rank, even if $\boldsymbol{\Phi}_{L}(\omega)$ does not.

\section{B. Multiple Channels}

In the single channel case, it was still necessary that the cutoff frequency $\Omega_{\mathrm{c}}$ of the LPF is at least $N$ times larger than the bandwidth of the signal $\widehat{a}$ in order to be able to recover the signal. Using several channels can reduce the cutoff frequency $\Omega_{\mathrm{c}}$ of the filter in each channel, from which the original signal $x$ is still recoverable. Suppose now that we have $M \geq N$ preprocessing channels, each of which uses a different mixing sequence, as in Fig. 2. Since $M \geq N$, we expect to be able to reduce the cutoff in each channel. We therefore consider the case in which $\Omega_{\mathrm{c}}=\Omega$. The output $y_{m}=\mathrm{P}_{\Omega_{\mathrm{c}}}\left(p_{m} x\right)$ of the $m$ th channel in the frequency domain is then equal to

$$
\widehat{y}_{m}(\omega)=\widehat{\gamma}_{m}(\omega)^{\mathrm{T}} \widehat{\mathbf{a}}\left(\mathrm{e}^{\mathrm{i} \omega T}\right), \quad \omega \in[-\Omega / 2, \Omega / 2]
$$

where $\widehat{\gamma}_{m}(\omega)$ is the length $N$ vector with $n$th element

$$
\left[\widehat{\gamma}_{m}(\omega)\right]_{n}=\widehat{\gamma}_{m, n}(\omega):=\sum_{k \in \mathbb{Z}} b_{k}^{(m)} \widehat{\phi}_{i}(\omega+k \Omega),
$$

and where $\left\{b_{k}^{(m)}\right\}_{k \in \mathbb{Z}}$ are the Fourier coefficients associated with the $m$ th sequence $p_{m}$. Defining by $\widehat{\mathbf{y}}(\omega)$ the length $M$ vector with $m$ th element $\widehat{y}_{m}(\omega)$, we conclude that

$$
\widehat{\mathbf{y}}(\omega)=\boldsymbol{\Gamma}_{M}(\omega) \widehat{\mathbf{a}}\left(\mathrm{e}^{\mathrm{i} \omega T}\right), \quad \omega \in[-\Omega / 2, \Omega / 2]
$$

where $\boldsymbol{\Gamma}_{M}(\omega)$ is the $M \times N$ matrix with entries $\left[\boldsymbol{\Gamma}_{M}(\omega)\right]_{m, n}=$ $\widehat{\gamma}_{m, n}(\omega)$. Now, all we need is to choose the $M$ sequences $\left\{b_{k}^{(m)}\right\}_{k \in \mathbb{Z}} \in \ell^{2}$ such that $\boldsymbol{\Gamma}_{M}(\omega)$ has full column rank. More specifically, as before we can write

$$
\boldsymbol{\Gamma}_{M}(\omega)=\mathbf{B}_{M} \boldsymbol{\Phi}(\omega),
$$

where $\mathbf{B}_{M}$ is a matrix with $M$ rows whose $m$ th row is given by the sequence $\left\{b_{k}^{(m)}\right\}_{k \in \mathbb{Z}}$, i.e.

$$
\left[\mathbf{B}_{M}\right]_{m, k}=b_{k}^{(m)}, \quad m=1, \ldots, M ; k \in \mathbb{Z} .
$$

By our assumption $\boldsymbol{\Phi}(\omega)$ has full column rank and so it remains to choose $\mathbf{B}_{M}$ such that $\boldsymbol{\Gamma}_{M}(\omega)$ is invertible for every $\omega \in[-\Omega / 2, \Omega / 2]$.

Note that compared with the previous section, where only one mixing sequence was applied, the problem of finding an appropriate matrix $\mathbf{B}_{M}$ becomes simpler: In the former case $\mathbf{B}_{L}$ has to have the special (diagonal) form (19), whereas the entries of $\mathbf{B}_{M}$ can be chosen almost arbitrarily.

\section{ApPliCATIONS AND EXAMPLES}

A special choice of periodic functions that are easy to implement in practice are binary sequences. These mixing functions were studied in [13] in the context of sparse multiband sampling. More specifically, $p_{m}, m=1, \ldots, M$ are chosen to attain the values \pm 1 over intervals of length $T / P$ where $P$ is a given integer. Formally,

$$
p_{m}(t)=\alpha_{n}^{(m)}, \quad n \frac{T}{P} \leq t<(n+1) \frac{T}{P}, \quad n=0, \ldots, P-1
$$

with $\alpha_{n}^{(m)} \in\{+1,-1\}$. In this case the Fourier coefficients (12) of the $m$ th sequence $p_{m}$ become

$$
b_{k}^{(m)}=\frac{1}{T} \sum_{n=0}^{P-1} \alpha_{n}^{(m)} \mathrm{e}^{-\mathrm{i} \frac{2 \pi}{P} n k} \int_{0}^{T / P} \mathrm{e}^{-\mathrm{i} \frac{2 \pi}{T} k t} \mathrm{~d} t .
$$

Setting $\omega_{0}:=2 \pi / P$ and evaluating the integral gives

$$
b_{0}^{(m)}=\frac{1}{P} \widehat{\alpha}_{0}^{(m)} \quad \text { and } \quad b_{k}^{(m)}=\frac{1-\mathrm{e}^{-\mathrm{i} \omega_{0} k}}{\mathrm{i} 2 \pi k} \widehat{\alpha}_{k}^{(m)}, \quad k \neq 0
$$

where $\left\{\widehat{\alpha}_{k}^{(m)}\right\}_{k \in \mathbb{Z}}$ denotes the $P$-periodic discrete Fourier transform (DFT) of the sequence $\left\{\alpha_{n}^{(m)}\right\}_{n=0}^{P-1}$. With these mixing sequences, the matrix $\mathbf{B}_{M}$ can be written as

$$
\mathbf{B}_{M}=\mathbf{Q F}^{*} \mathbf{W}
$$

where $\mathbf{Q}$ is a matrix with $P$ columns and $M$ rows, whose $m$ th row is given by the sequence $\left\{\widehat{\alpha}_{n}^{(m)}\right\}_{n=0}^{P-1}, \mathbf{F}$ is the $P \times P$ Fourier matrix, and $\mathbf{W}$ is a matrix with $P$ rows and infinitely many columns consisting of block diagonal matrices of size $P \times P$ whose diagonal values are given by the sequence $\left\{w_{k}\right\}_{k \in \mathbb{Z}}$ defined by $w_{0}=1 / P$ and $w_{k}=\frac{1-\mathrm{e}^{-\mathrm{i} \omega_{0} k}}{\mathrm{i} 2 \pi k}$ for $k \neq 0$. Applying these binary mixing sequences, the problem is now to find a finite $M \times P$ matrix $\mathrm{Q}$ with values in $\{+1,-1\}$ such that $\mathbf{Q F}^{*} \mathbf{W} \boldsymbol{\Phi}(\omega)$ has full column rank for every $\omega \in[-\Omega / 2, \Omega / 2]$. The next example shows how to select $\mathbf{Q}$ in the case of bandlimited generators.

EXAMPLE 1 (BANDLIMITED GENERATORS): We consider the case where each generator $\phi_{n}$ is bandlimited to the interval $[-K \Omega / 2, K \Omega / 2]$ for some $K \in \mathbb{N}$, and with $N=2 K+1$ generators. In this case, $\boldsymbol{\Phi}(\omega)=\boldsymbol{\Phi}_{K}(\omega)$ is essentially an $N \times N$ matrix (all other entries are zero) which is invertible for every $\omega \in[-\Omega / 2, \Omega / 2]$ according to assumption (4).

We now apply $M=N$ different mixing sequences $\left\{p_{m}\right\}_{m=1}^{M}$ having the special structure (21), and we choose $P=N$. According to (20) and (22) the matrix $\Gamma_{M}(\omega)$ then becomes

$$
\Gamma_{M}(\omega)=\mathbf{Q} \mathbf{F}^{*} \mathbf{W} \boldsymbol{\Phi}(\omega),
$$

where $\mathbf{Q} \mathbf{F}^{*}$ and $\mathbf{W} \boldsymbol{\Phi}(\omega)$ are matrices of size $N \times N$. The matrix $\mathbf{W} \Phi(\omega)$ may be considered as the product of the invertible $N \times N$ matrix $\boldsymbol{\Phi}(\omega)=\boldsymbol{\Phi}_{K}(\omega)$ with an $N \times N$ diagonal matrix consisting of the central diagonal matrix of $\mathbf{W}$, i.e.

$$
\mathbf{W} \boldsymbol{\Phi}(\omega)=\operatorname{diag}\left(w_{0}, \ldots, w_{N-1}\right) \boldsymbol{\Phi}_{K}(\omega)
$$


Since this diagonal matrix is invertible also $\mathbf{W} \boldsymbol{\Phi}_{K}(\omega)$ is invertible for every $\omega \in[-\Omega / 2, \Omega / 2]$. Therefore, using the fact that the Fourier matrix $\mathbf{F}$ is invertible, $\Gamma_{M}(\omega)$ is invertible for each $\omega \in[-\Omega / 2, \Omega / 2]$ if the values $\left\{\alpha_{n}^{(m)}\right\}_{n=1}^{P}$ of the mixing sequences $p_{m}$ are chosen such that $\mathbf{Q}$ is invertible. This can be achieved by choosing $\mathbf{Q}$ as a Hadamard matrix of order $N$. It is known that Hadamard matrices exists at least for all orders up to 667 [14].

In the previous example, $\boldsymbol{\Phi}_{K}(\omega)$ was an $N \times N$ invertible matrix for all $\omega \in[-\Omega / 2, \Omega / 2]$. According to Prop. 2 a recovery of the signal $x$ without preprocessing is therefore possible if the bandwidth $\Omega_{\mathrm{c}}$ of the LPF is larger than $N \Omega$. However, the previous example shows that preprocessing of $x$ by applying binary sequences in $M=N$ channels allows recovery of the signal already from its signal components in the frequency range $[-\Omega / 2, \Omega / 2]$.

For simplicity we assumed throughout that the LPF bandwidth $\Omega_{\mathrm{c}}$ is equal to the signal bandwidth $\Omega$ and that the number of channels $M$ is at least equal to the number of generators $N$. However, if $M<N$, recovery of the signal may still be possible if the bandwidth of the LPF is increased.

\section{Connection With Sparse Analog Signals}

In this section we want to discuss the incorporation of sparsity into our approach. To this end, we follow [5] to describe sparsity of analog signals in SI spaces. Specifically, we assume that only $K$ out of the $N$ generators $\phi_{n}$ are active, so that at most $K$ of the sequences $\left\{a_{n}[k]\right\}_{k \in \mathbb{Z}}$ are nonzero.

In [5], it was shown how such signals can be sampled and reconstructed from samples at a low rate of $2 K / T$. The samples are obtained by pre-processing the signal $x$ with a set of $2 K$ sampling filters, whose outputs are uniformly sampled at a rate of $1 / T$. Without the sparsity assumption, at least $N$ sampling filters are needed where generally $N$ is much larger than $K$. In contrast to this setup, here we are constrained to sample at the output of a LPF with given bandwidth. Thus, we no longer have the freedom to choose the sampling filters as we wish. Nonetheless, by exploiting the sparsity of the signal we expect to be able to reduce the bandwidth needed to recover $x(t)$ of the form (1), or in turn, to reduce the number of branches needed when using a bank of modulators.

We have seen that the ability to recover $x$ depends on the left invertibility of the matrix $\boldsymbol{\Phi}_{L}(\omega)$ (or $\boldsymbol{\Gamma}_{L}(\omega)$ ). With appropriate definitions, our problem becomes that of recovering $\widehat{\mathbf{a}}\left(\mathrm{e}^{\mathrm{i} \omega T}\right)$ from the linear set of equations (10) (with $\Gamma_{L}(\omega)$ replacing $\boldsymbol{\Phi}_{L}(\omega)$ when preprocessing is used). Our definition of analog sparsity implies that at most $K$ of the Fourier transforms $\widehat{a}_{n}(\omega)$ have non-zero energy. Therefore, the infinite set of vectors $\left\{\widehat{\mathbf{a}}\left(\mathrm{e}^{\mathrm{i} \omega T}\right), \omega \in[-\Omega / 2, \Omega / 2]\right\}$ share a joint sparsity pattern with at most $K$ rows that are not zero. This in turn allows us to recover $\left\{\widehat{\mathbf{a}}\left(\mathrm{e}^{\mathrm{i} \omega T}\right), \omega \in[-\Omega / 2, \Omega / 2]\right\}$ from fewer measurements. Under appropriate conditions, it is sufficient that $\widehat{\mathbf{y}}(\omega)$ has length $2 K$, which in general is much smaller than $N$. Thus, fewer measurements are needed with respect to the full model (1). The reduction in the number of mea- surements corresponds to choosing a smaller bandwidth of the $\mathrm{LPF}$, or reducing the number of modulators.

In order to recover the sequences in practice, we rely on the separation idea advocated in [4]: we first determine the support set, namely the active generators. This can be done by solving a finite dimensional optimization problem under the condition that $\boldsymbol{\Phi}_{L}(\omega)$ (or $\boldsymbol{\Gamma}_{L}(\omega)$ ) are fixed in frequency up to a possible frequency-dependent normalization sequence. Recovery is then obtained by applying results regarding infinite measurement vector (IMV) models [4]. When $\boldsymbol{\Phi}_{L}(\omega)$ does not satisfy this constraint, we can still convert the problem to a finite dimensional optimization problem as long as the sequences $\left\{a_{n}[k]\right\}$ are rich [6]. This implies that every finite set of vectors share the same frequency support. We refer to [4]-[6] for more details on the recovery of space signals.

The main point we want to stress is that the ideas developed in this paper can also be used to treat the recovery of sparse SI signals from their lowpass content. The difference is that we can relax the requirement for invertibility of $\boldsymbol{\Phi}_{L}(\omega), \boldsymbol{\Gamma}_{L}(\omega)$. Instead, it is sufficient that these matrices satisfy the known conditions from the compressed sensing literature. This in turn allows in general reduction of the LPF bandwidth, or the number of modulators, in comparison with the non-sparse scenario.

\section{REFERENCES}

[1] C. de Boor, R. DeVore, and A. Ron, "The structure of finitely generated shift-invariant spaces in $L^{2}\left(\mathbb{R}^{d}\right)$, , J. Funct. Anal., vol. 119, no. 1, pp. 37-78, 1994.

[2] J. S. Geronimo, D. P. Hardin, and P. R. Massopust, "Fractal functions and wavelet expansions based on several scaling functions," J. Approx. Theory, vol. 78, no. 3, pp. 373-401, 1994.

[3] O. Christensen and Y. C. Eldar, "Generalized shift-invariant systems and frames for subspaces," J. Fourier Anal. Appl., vol. 11, no. 3, pp. 299-313, Jun. 2005

[4] M. Mishali and Y. C. Eldar, "Reduce and Boost: Recovering Arbitrary Sets of Jointly Sparse Vectors," IEEE Trans. Signal Process., vol. 56, no. 10, pp. 4692-4702, Oct. 2008.

[5] Y. C. Eldar, "Compressed sensing of analog signals in shift-invariant spaces," IEEE Trans. Signal Process., vol. 57, no. 8, pp. 2986-2997, Aug. 2009.

[6] _- "Uncertainty Relations for Shift-Invariant Analog Signals," IEEE Trans. Inf. Theory, vol. 55, no. 12, pp. 5742-5757, Dec. 2009.

[7] K. Gedalyahu and Y. C. Eldar, "Time-Delay Estimation From LowRate Samples: A Union of Subspaces Approach," IEEE Trans. Signal Process., vol. 58, no. 6, pp. 3017-3031, Jun. 2010.

[8] Y. C. Eldar and V. Pohl, "Recovering Signals from Lowpass Data," IEEE Trans. Signal Process., vol. 58, no. 5, pp. 2636-2646, May 2010.

[9] M. Vetterli, P. Marziliano, and T. Blu, "Sampling Signals With Finite Rate of Innovation," IEEE Trans. Signal Process., vol. 50, no. 6, pp. 1417-1428, Jun. 2002.

[10] M. Mishali and Y. C. Eldar, "Blind Multiband Signal Reconstruction: Compressed Sensing for Analog Signals," vol. 57, no. 3, pp. 993-1009, Mar. 2009.

[11] I. J. Schoenberg, Cardinal Spline Interpolation. Philadelphia, PA: SIAM, 1973.

[12] Y. C. Eldar and T. Michaeli, "Beyond bandlimited sampling: Nonlinearities, smoothness and sparsity," IEEE Signal Process. Mag., vol. 26, no. 3, pp. 48-68, May 2009.

[13] M. Mishali and Y. C. Eldar, "From Theory to Practice: Sub-Nyquist Sampling of Sparse Wideband Analog Signals," IEEE J. Sel. Topics Signal Process., vol. 4, no. 2, pp. 375-391, Apr. 2010.

[14] H. Kharaghani and B. Tayfeh-Rezaie, "A Hadamard matrix of order 428," J. Combin. Des., vol. 13, no. 6, pp. 435-440, Nov. 2005. 